\title{
Note
}

\section{$\alpha$-Glucosidase Inhibitory Activity in Leaves of Some Mulberry Varieties}

\author{
Kazuhisa Yatsunami, ${ }^{1}$ Youichi SAITO, ${ }^{1}$ Eiichi FuKUdA, ${ }^{1}$ Satoshi ONODERA ${ }^{2}$ and Kengo Oshigane ${ }^{3}$ \\ ${ }^{1}$ Faculty of Agriculture, Tamagawa University, 6-1-1 Tamagawa-gakuenn, Machida, Tokyo 194-8610, Japan \\ ${ }^{2}$ Showa Pharmaceutical University, 3-3165 Higashi-tamagawa-gakuenn, Machida, Tokyo 194-8543, Japan \\ ${ }^{3}$ Faculty of Engineering, Soka University, 1-236 Tangityo, Hachioji, Tokyo 192-8577, Japan
}

Received June 18, 2003; Accepted August 28, 2003

\begin{abstract}
The seasonal and polyploidal changes of $\alpha$-glucosidase (rat-intestinal) inhibitory activity in mulberry leaves were compared in 12 mulberry varieties. Enashi, Fusoumaru, No. 325 and Kenmochi which are classified as 3 major varieties in Japan were harvested in May or August as the field samples. Shimaguwa and the 3 different wild types such as Kuromiguwa, Nagamiguwa, Midoriguwa were harvested in May as the greenhouse samples. Some varieties harvested in the field or greenhouse indicated a difference in $\alpha$-glucosidase. In the field Enashi was strong, while Kenmochi was rather weak. The seasonal changes of inhibition between May and August were differed among the varieties. The $3 x$ variety in the field or greenhouse showed the strong inhibition within the same variety.
\end{abstract}

Keywords: $\alpha$-glucosidase, inhibitor, mulberry, Morus, polyploidy, variety

Mulberry leaves (Morus alba L.) have been used as a traditional medicine with anti-inflammatory and anti-hyperglycemic action (Kimura et al., 1995). The mulberry leaf component such as 1-deoxynojirimycin (DNJ; Yoshikuni, 1988; Asano et al., 1994), 2-arylbenzofuran derivatives (Basnet et al., 1993) and polyhydroxylated alkaloids (Asano et al., 2001) inhibit the activity of $\alpha$-glucosidase of the small intestine, and also prevent sudden hydrolysis of disaccharides, absorption of glucose into the blood vessel, and resultant increase of blood glucose level. DNJ showed stronger inhibitory activity towards rat intestinal maltase and yeast $\alpha$-glucosidase compared with DNJ-trehalamine-fused and -linked compounds (Shiozaki et al., 1998). The extract derived from mulberry leaves potentiated the pilocarpine-induced salivary flow which was correlated with antihyperglycemic effects (Chen et al., 1995). Fagomine from mulberry leaves increased the plasma insulin level in STZ-diabetic mice, and this insulin release by fagomine might partly contribute to the antihyperglycemic action (Nojima et al., 1998; Taniguchi et al., 1998). The maximum reduction in blood glucose of leaf extract was ob-served among fruit, trunk bark and root bark (Hosseinzadeh \& Sadeghi, 1999).

On the other hand, there are many reports concerning artificially polyploidy from the point of view of cytology (Seki \& Oshigane, 1953, 1957, 1959; Tojyo, 1954, 1963, 1966; Das \& Krishnaswami, 1964; Das et al., 1970) as mulberry breeding is extremely valuable for the sericulture. It is well accepted that major characteristics of 3,4x polyploidy are indicated a large leaf, high saccharide content, high yield and high feed efficiency on silkworm among the different polyploidy. We observed that the changes of $\alpha$-glucosidase inhibitory activity in leaves were occurred among mulberry polyploidy. Activity for lowering blood-glucose levels of autumn-rearing silkworm was higher

\footnotetext{
* To whom correspondence should be addressed.
}

E-mail: yatsunam@agr.tamagawa.ac.jp than that of spring-rearing silkworm (Ryu et al., 1997). The changes in $\alpha$-glucosidase inhibitory activity in leaves can be observed in some mulberry varieties, there is no reports concerning of seasonal or varietal changes on the $\alpha$-glucosidase inhibition in leaves of any mulberry variety.

In this study the seasonal and polyploidal changes of $\alpha$-glucosidase inhibition in mulberry leaves were compared in 12 mulberry varieties which included 3 major varieties and wild type species.

\section{Materials and Methods}

Materials The mulberry varieties used in this study were 3 major families (Yamaguwa, Karayamaguwa and Roguwa family) and 3 kinds of wild type. Kenmochi (M.bombycis Koidz. 2, 3, 4x) belonging to the Yamaguwa family. No. 325 (M. alba L. 3x) and Enashi (M. alba L. form. sessifolia Hotta 2, 3x) belonging to the Karayamaguwa family and Fusoumaru (M. latifolia poiret $2 \mathrm{x}$ ) belonging to the Roguwa family. Simaguwa (M. asidosa Griff 2, 3, 4x) belonged to the Shimaguwa family. Midoriguwa (M. wittiorum Hand.-Maz. 4x), Nagamiguwa (M. laevigata Wall 3x from China or India) and Kuromiguwa (M. nigra L. 22x from Lebanon) were wild type. These mulberry leaves (Enashi, Fusoumaru, No. 325, Kenmochi) were harvested May 2, and August 8, 2002 from the mulberry field of Soka University. The potted mulberry (Midoriguwa, Kuromiguwa, Shimaguwa, Nagamiguwa) were harvested May 30, 2002 from the greenhouse in Soka University. The leaves harvested were stored in a polyethylene bag in the freezer at $-20^{\circ} \mathrm{C}$.

Preparation of the sample solution Five grams of mulberry leaves were homogenized with $40 \mathrm{ml}$ of distilled water for 3 min at $10,000 \mathrm{rpm}$. The homogenate was centrifuged at 3000 rpm for $10 \mathrm{~min}$. The supernatant was obtained by filtration and the precipitate was suspended with $30 \mathrm{ml}$ of distilled water and centrifuged again. The supernatant was filtered and combined with the first supernatant. This manipulation was repeated one 


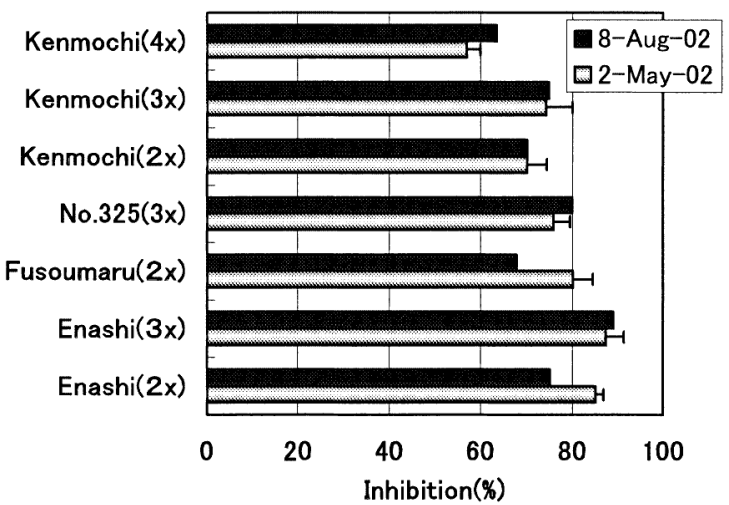

Fig. 1. $\alpha$-Glucosidase inhibitory activity of mulberry leaves in the field harvested in May and August.

more time, then the combined supernatants were filled up to 100 $\mathrm{ml}$ with distilled water and used for sample solution.

Measurement of $\alpha$-glucosidase inhibitory activity One gram of rat-intestinal acetone powder was suspended in $10 \mathrm{ml}$ of $0.9 \%$ saline, and the suspension sonicated $(1 \mathrm{~min} \times 3)$. After centrifugation $(3000 \mathrm{rpm} \times 30 \mathrm{~min})$, the resulting supernatant was diluted 4 times with distilled water and used for the assay as enzyme solution. The assay mixture consisted of a $100 \mathrm{~mm}$ maleate buffer ( $\mathrm{pH} 6.0,0.7 \mathrm{ml}), 500 \mathrm{mM}$ maltose $(0.1 \mathrm{ml})$, and the sample solution; the mixture was preincubated for $5 \mathrm{~min}$ at $37^{\circ} \mathrm{C}$. The reaction was initiated by adding a crude $\alpha$-glucosidase solution $(0.1 \mathrm{ml})$ to the reaction mixture; this mixture was then incubated for $60 \mathrm{~min}$ at $37^{\circ} \mathrm{C}$, the reaction being terminated by adding $1.0 \mathrm{ml}$ of a $2.0 \mathrm{M}$ maleate-Tris-NaOH buffer ( $\mathrm{pH}$ 7.4). The glucose released in the reaction mixture was determined by a Glucose C-II Test Wako kit (Wako Pure Chemical Co., Tokyo) (Ohta et al., 2002). The significantly difference of each of the two groups was surveyed with a $p$ value by Student's $t$-test assuming that the dispersion of both the two groups was not equal.

\section{Results and Discussion}

Comparison of the inhibition with variety in the field Figure 1 shows the inhibitory activity in the field. In spring or summer Enashi and No. 325 which were classified in the Karayamaguwa family showed strong inhibition, while Kenmochi in the Yamaguwa family showed rather weak inhibition. The inhibition of $3 \mathrm{x}$ variety was indicated strong inhibition compared to that of $2 \mathrm{x}$ or $4 \mathrm{x}$. In $3 \mathrm{x}$ varieties Enashi was highest and second was No. 325 , the third was Kenmochi. In $2 x$ varieties a significant difference was not seen. Comparison between May and August Fusoumaru and Kenmochi (4x) showed significant $(p<$ 0.05 ) difference. Fusomaru was strong in May and Kenmochi was strong in August. In other varieties no significant seasonal difference existed in the field. As Enashi was a member of the Karayamaguwa family, other $3 x$ varieties in this family such as Shinkenmochi and Akagi might also have strong inhibition. In this observation it was indicated that Enashi (3x) which is one of the major families was very valuable as an ingredient for health food or a supplement combined with mulberry leaves.

Comparison of the inhibition with the variety in the greenhouse Figure 2 shows the inhibitory activity of the Shimagawa

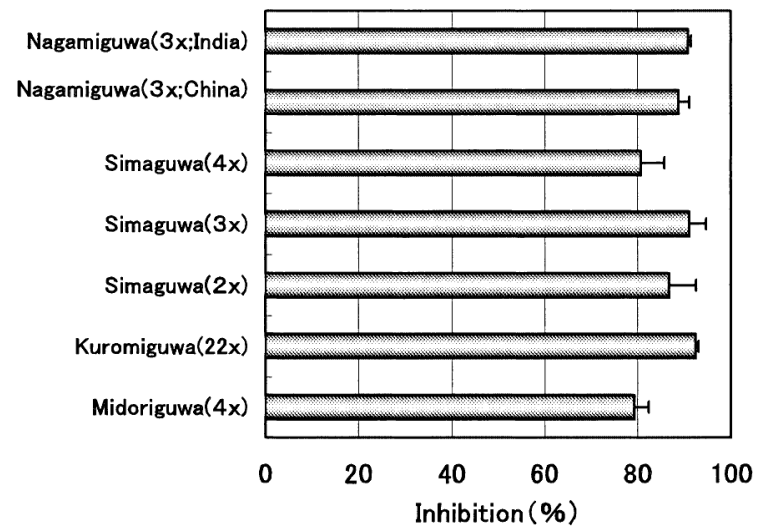

Fig. 2. $\alpha$-Glucosidase inhibitory activity of mulberry leaves in the greenhouse harvested in May.

family and the wild type in the greenhouse. In the Shimagawa family $3 \mathrm{x}$ was the strongest, in wild type Kuromiguwa (22x) was the strongest. In this observation the inhibition of $3 x$ variety was also showed strong except for $22 \mathrm{x}$ compared with that of $2 \mathrm{x}, 4 \mathrm{x}$. There was no significant difference between Nagamiguwa (China) and Nagamiguwa (India).

From these results, some varieties harvested in spring or summer showed a difference in $\alpha$-glucosidase. Enashi (3x) was strong among the field samples and Kuromiguwa (22x), Shimaguwa (3x) and Nagamiguwa (3x) were strong among the greenhouse samples. The seasonal changes of inhibition between May and August differed among the varieties. $3 \mathrm{x}$ variety showed strong inhibition within the same variety. Thus, the seasonal and polyploidal differences in inhibition for some varieties were observed, but seen within our observation, further observation on more other kinds of mulberry varieties should be required. It is assumed that the differences in inhibition activities in leaves is caused by the differences in DNJ contents. We plan in the near future to study the relation between the inhibitory activities and DNJ contents in leaves of some mulberry varieties.

\section{References}

Asano, N., Tomioka, E., Kizu, H. and Matsui, K. (1994). Sugars with nitrogen in the ring isolated from the leaves of Morus bombycis. Carbohydr. Res., 253, 235-245.

Asano, N., Yamashita, T., Yasuda, K., Ikeda, K., Kizu, H., Komeda, Y., Kato, A., Nash, R.J., Lee, H.S. and Ryu, K.S. (2001). Polyhydroxylated alkaloids isolated from mulberry trees (Morus alba L.) and silkworms (Bombyx mori L.). J. Agric. Food. Chem., 49, 42084213.

Basnet, P., Kadota, K., Terashima, S., Shimizu, M. and Namba, T. (1993). Two new 2-arylbenzofuran derivatives from hypoglycemic activity-bearing fractions of Morus insignis. Chem. Pharm. Bull., 41, 1238-1243.

Chen, F.J., Nakashima, N., Kimura, I., Kimura, M., Asano, N. and Koya, S. (1995). Potentiating effects on pilocarpine-induced saliva secretion, by extracts and N-containing sugars derived from mulberry leaves. Biol. Pharm. Bull., 18, 1676-1680.

Das, B.C. and Krishnaswami, S. (1964). Effect of colchicine on germinating seeds of mulberry. Indian J. Seric., 3, 5-6.

Das, B.C., Prasad, D.N. and Sikdar, A.K. (1970). Colchicine induced tetraploids of mulberry. Caryologia, 23, 283-293.

Hosseinzadeh, H. and Sadeghi, A. (1999). Antihyperglycemic effects of Morus nigra and Morus alba in mice. Pharm. Pharmacol. Lett., 9, 63-65. 
Kimura, M., Chen, F.J., Nakashima, N., Kimura, I., Asano, N. and Koya, S. (1995). Antihyperglycemic effects of N-containing sugars derived from mulberry leaves in streptozocin-induced diabetic mice. J. Tradit. Med., 12, 214-219.

Nojima, H., Kimura, I., Chen, F., Sugihara, Y. and Haruno, M. (1998). Antihyperglycemic effects of N-containing sugars from Xanthocercis zambesiaca, Morus bombycis, Aglaonema treubii, and Castanospermum australe in Streptozotocin-diabetic mice. J. Nat. Prod., 61, 397-400.

Ohta, T., Sasaki, S., Oohori, T., Yoshikawa, S. and Kurihara, H. (2002). $\alpha$-Glucosidase inhibitory activity of a $70 \%$ methanol extract from Ezoishige (Pelvetia babingtonii de Toni) and its effect on the elevation of blood glucose level in rats. Biosci. Biotechnol. Biochem., 66, 1552-1554.

Seki, H. and Oshigane, K. (1953). Studies in polyploid mulberry trees (I). Tetraploid mulberry trees induced by colchicine treatment. Fac. Text. Seric. Shinsyu Univ., 3, 11-17.

Seki, H. and Oshigane, K. (1957). Studies in polyploid mulberry trees (II). On the morphology of mixoploid mulberry trees induced by colchicine treatment. Fac. Text. Seric. Shinshu Univ., 7, 5-17.

Seki, H. and Oshigane, K. (1959). Studies in polyploid mulberry trees (III). The valuation of breeded polyploid mulberry leaves and the results of feeding silkworms on them. Fac. Text. Seric. Shinshu Univ., 9, 6-15.

Ryu, K.S., Lee, H.S., Chung, S.H. and Kang, P.D. (1997). An activity of lowering blood-glucose levels according to preparative conditions of silkworm powder. Korean J. Seric. Sci., 39, 79-85.

Shiozaki, M., Yoshiike, R., Ando, O., Ubukata, O. and Haruyama, H. (1998). Syntheses of 1-deoxynojirimycin-trehalamine-fused and -linked compounds and their biological activities. Tetrahedron, 54, 15167-15182.

Taniguchi, S., Asano, N., Tomino, F. and Miwa, I. (1998). Potentiation of glucose-induced insulin secretion by fagomine, a pseudo-sugar isolated from mulberry leaves. Horm. Metab. Res., 30, 679-683.

Tojyo, I. (1954). Tetraploid of the mulberry tree produced by the Colchicine method. J. Seric. Jpn., 23, 242.

Tojyo, I. (1963). Autotetraploid indued by colchicine treatment in mulberry seedling. J. Seric. Jpn., 32, 34-37.

Tojyo, I. (1966). Studies on polyploid mulberry tree (I). Breeding of artificial autotetraploids. Bulel. Seric. Exp. Sta. Jpn., 20, 187-207.

Yoshikuni, Y. (1988). Inhibition of intestinal $\alpha$-glucosidase activity and postprandial hyperglycemia by Moranoline and its $\mathrm{N}$-alkyl derivatives. Agric. Biol. Chem., 52, 121-128. 